\title{
Exploring Diminished Reality (DR) Spaces to Augment the Attention of Individuals with Autism
}

\section{Conference Paper}

\section{Author(s):}

Yantaç, Asım; Çorlu, Doğa; Fjeld, Morten; Kunz, Andreas (D)

Publication date:

2015

Permanent link:

https://doi.org/10.3929/ethz-a-010565943

Rights / license:

In Copyright - Non-Commercial Use Permitted

Originally published in:

https://doi.org/10.1109/ISMARW.2015.21 


\section{Exploring Diminished Reality (DR) Spaces to Augment the Attention of Individuals with Autism}

\author{
Asım Evren Yantaç \\ KUAR, \\ Koç University
}

\author{
Doğa Çorlu \\ KUAR, \\ Koç University
}

\author{
Morten Fjeld \\ T2I Lab, \\ Chalmers University of Technology
}

Andreas Kunz
ETH Zurich

workshops we conducted. These explorations led us to conceptual ideas such as a mirror-like interactive wall for filtering information, which is as yet an unexplored format for AUI research (Fig. 1), or augmenting the environment by taking advantage of projection mapping technologies. This paper presents expected future applications, and discusses some human factors of Diminished Reality. approach, we believe that AUIs may benefit attention disord challenges of individuals with autism spectrum disorder (ASD), who are adversely affected by continuously changing surroundings or distracting visual incidents even when no technologies are involved. With this perspective, we carried out eight participative design workshops with interaction designers and ASD domain experts aiming to explore possible AUI solutions for ASD individuals. Interestingly, these studies yielded a novel AUI concept consisting of a mirror-like wall interface filtering out irrelevant visual information from real-time capture of a space. This paper presents some future applications of DR and discusses related human factors. A contribution of this paper is early insights from the set of eight workshops.

\section{Author Keywords}

Diminished Reality (DR), Autism Spectrum Disorder (ASD), Attentive User Interface (AUI), attention disorder, augmented attention.

\section{INTRODUCTION}

Emerging technologies increase distraction due to the high number of displaying surfaces and increased amounts of data around us. In our everyday lives, we encounter many distracting factors. Pointing out the relevance of attentive user interfaces (AUIs) by referring to the fact that our attention is a limited resource, Vertegaal [24] claims that we may better manage our attention by using technologies sensing, analyzing, and keeping track of our surroundings and ourselves. In this sense, AUIs are seen as systems tracking factors in the environment such as proximity, orientation, or verbal communication. Some of these studies follow a Diminished Reality (DR) approach for filtering out the information we receive before it reaches our sensory system. While most AUI solutions have been intended for HCI, recently it has also started to be addressed for human-human interaction (HHI) as well. These advances provide some playful and experimental novel opportunities for interaction design.

With a similar approach, we propose here that AUI solutions may benefit general attention-related disorders, such as in autism spectrum disorder (ASD). ASD is defined as a pervasive, lifelong developmental disorder that appears early in an individual's life. It impedes the individual's social interaction and communication; partly due to reduced ability to comprehend and express emotions, and partly to high sensitivity towards physical stimuli such as sound, proximity, and changing surroundings.

Our experimental idea of diminishing the reality for augmented attention offers the possibility of optimizing the particular sensory system operating in our everyday life even when no technologies are involved. Here, we share our insights from our initial concept development studies of the literature review and eight design

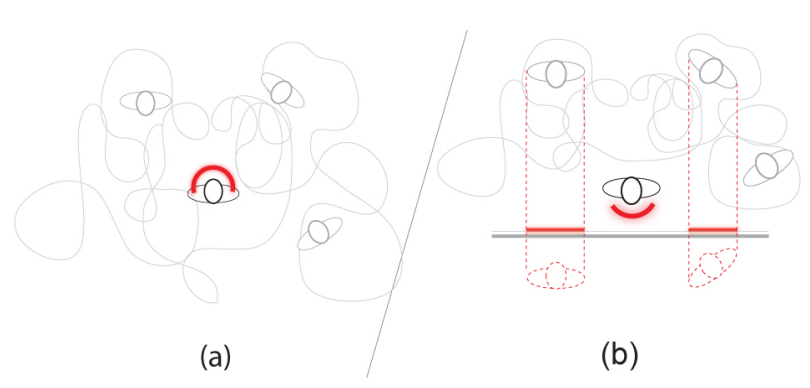

Figure 1: Diminished Reality (DR) concept: (a) the distracting environment that ASD individual facing (b) mirror-like interactive wall filtering out irrelevant information from distracting environment

\section{BACKGROUND}

As already mentioned, HCI researchers have investigated intelligent technologies for improved work performance with a special interest in human factors. Besides a focus on sensory and attention processes, we see an opportunity to make interactive devices smart enough to watch our needs and provide information whenever necessary. Human factor research has been dealing with this problem over the last decade under the name of attentive user interfaces (AUI). Human factors and disabling environment design are broad subjects with many alternative approaches. Here, we focus on the following three research issues: (a) Filtering from the general subject of AUIs; (b) augmenting users' visual attention, and (c) interactive assistive technologies for individuals with attention disorders.

\section{Attentive User Interfaces}

An early AUI study by Vertegaal [24] refers to the groundbreaking motto of the famous architect Mies Van Der Rohe, "less is more" to explain the need for smart interactive devices observing and communicating our attention in an economic way. For mediating and refining sound before it reaches our brain, literature has several different solutions such as smart Liebl et al. [15], visual distraction has been surprisingly unexplored until the last decade. Recently though, the topic seems to have reappeared within human factor and work performance studies $[6,15,22]$. Refining visual distractions is needed in any work-related task because it affects reasoning, searching and detection abilities directly. Most eye-tracking research studies and scenarios focus on tasks related to visual search and detection of objects. headphones [2] or transparent hearing [16]. But as mentioned by 
Narayanan and Yoon [17] defined five (a-e) characteristic settings with visual, spatial, and causal reasoning tasks where attentive displays would be useful. These are where: (a) objects of the domain are spatially distributed; (b) the domain is dynamic; (c) objects causally interact with each other; (d) such interactions can be traced along chains of cause-effect relationships that branch and merge in spatial and temporal dimensions; and (e) predicting the future evolution of a system in the domain requires reasoning from a given set of initial conditions and inferring these causal chains of events. Mapping the five AUI characteristic settings [17] with the AS disorder situations is part of the concept we suggest here (Table 1).

\begin{tabular}{|l|l|}
\hline AUI settings [17] & ASD situation \\
\hline $\begin{array}{l}\text { (a) Objects are spatially } \\
\text { distributed }\end{array}$ & $\begin{array}{l}\text { We consider the work } \\
\text { environment in which task } \\
\text { related objects are distributed. }\end{array}$ \\
\hline (b) Domain is dynamic & $\begin{array}{l}\text { ASD individual is mostly } \\
\text { dynamic in his work } \\
\text { environment. }\end{array}$ \\
\hline $\begin{array}{l}\text { (c) Objects causally interact } \\
\text { with each other }\end{array}$ & $\begin{array}{l}\text { There is continuous interaction } \\
\text { between the domain and human } \\
\text { or computers in the } \\
\text { surroundings. }\end{array}$ \\
\hline $\begin{array}{l}\text { (d) Interactions along } \\
\text { cause-effect relationships }\end{array}$ & $\begin{array}{l}\text { Work related tasks need } \\
\text { reasoning and ASD individuals } \\
\text { lack reasoning abilities. }\end{array}$ \\
\hline $\begin{array}{l}\text { (e) Reasoning from a given } \\
\text { set of initial conditions }\end{array}$ & $\begin{array}{l}\text { Personal abilities and } \\
\text { preferences of the ASD } \\
\text { individual should be known and } \\
\text { entered as predefined } \\
\text { knowledge. ASD would not } \\
\text { accept surprises. }\end{array}$ \\
\hline
\end{tabular}

Table 1: Mapping of situations calling for AUI solutions listed by Narayanan and Yoon [17] (left column) with results from our Autism Spectrum Disorder (ASD) case study (right column)

From another perspective, Shell et al. [22] listed five key functions of AUIs, which are: (i) sensing attention; (ii) reasoning about attention; (iii) regulating interactions; (iv) communicating attention; (v) augmenting attention (Table 2). Looking at the related work with the intention on these functions; we can see eyecontingent [19, 25], bio-feedback [4], social geometry or proximity [11] solutions for sensing the attention, which has been the most popular focus until now. Even though these AUI functions and case studies or techniques have common interests with our case study, our focus is more related to the communication and augmentation of visual attention. We will next consider related works concerning augmented visual attention.

\section{Augmenting Visual Attention}

As listed above, there are many reasons for augmenting visual attention. For example, it might be needed when dealing with large sets of information using small displays, or when there is a huge display in front but focus on specific content is needed. Toet [25] points out that applications of such technologies are related to critical work fields such as flight, medicine, driving simulation, virtual reality, remote piloting and tele-operation, infrared and indirect vision, image transmission and retrieval, telemedicine video tele-conferencing and artificial vision systems. His detailed overview categorizes the visual dynamic information display techniques to enhance visual capacity by refining the view or content according to the limitations of the user; (a) Non-distortion oriented techniques; (b) Distortion-oriented techniques; (c) Hybrid techniques: magic lens filters; (d) Multiple screens; (e) Gaze contingent multi-resolution displays; (f) Gaze contingent stereoscopic displays; (g) Gaze contingent attention guiding displays. As already mentioned, within these techniques gazecontingent solutions have been the most popular, as an extension of the emerging interest in eye tracking in which the system tracks gaze and reduces the detail of the image left out of focus [18]. This subject is also relevant to eye-contingent AUIs and covert attention, and deals with the level of information needed within the fovea. Besides the help of augmenting attention, this solution is also used to reduce the bandwidth demands for image transmission.

\begin{tabular}{|l|l|}
\hline AUI functions [22] & ASD case study focus \\
\hline (i) Sensing attention & $\begin{array}{l}\text { In the case of ASD, sensing } \\
\text { attention is a very detailed } \\
\text { critical issue which should } \\
\text { consider initial conditions, } \\
\text { personal abilities, preferences, } \\
\text { proximity and other inputs. It } \\
\text { is also closely related to } \\
\text { reasoning about attention. }\end{array}$ \\
\hline (ii) Reasoning about attention & $\begin{array}{l}\text { Reasoning is another critical } \\
\text { subject, which should be } \\
\text { considered alongside focus on } \\
\text { physiological parameters. We } \\
\text { do not cover these functions in } \\
\text { our research. }\end{array}$ \\
\hline (iii) Regulating interactions & $\begin{array}{l}\text { Regulation interactions will be } \\
\text { considered as an end result of } \\
\text { this study in the future. }\end{array}$ \\
\hline (iv) Communicating attention & $\begin{array}{l}\text { Communication of attention } \\
\text { will be considered in terms of } \\
\text { language used for refining the } \\
\text { visual message. }\end{array}$ \\
\hline (v) Augmenting attention & $\begin{array}{l}\text { Augmenting visual attention is } \\
\text { the main aim in our study. }\end{array}$ \\
\hline
\end{tabular}

Table 2: Mapping of AUI functions by Shell [22] (left column) and the scope of our study (right column)

What was not listed by Toet [25] though, were techniques for refining and augmenting visual attention after sensing with other means, for example, given sets of initial conditions or gazeindependent solutions like proximity. These are important issues especially when it comes to the office environment. We are only aware of a handful of recent works, such as EyePliances [22], Attentive Cubicle System [5], and Kinected Conference [7]. The Attentive Cubicle System regulates visual and auditory communication between employees in a cubicle layout office environment by watching their orientation and hiding or showing each other according to interests [5]. EyePlainces similarly watches employees' attention to regulate communication, while DeVincenzi et al. explore synthetic focusing techniques for enhancing video tele-conferences [7]. Finally, Skaburskis et al. [23] employ users' eye-gaze and orientation in front of a mirrorlike screen to communicate and augment their attention by adding virtual bubbles to their face. We tried to map the AUI functions listed above with current assistive solutions for attention disorder 


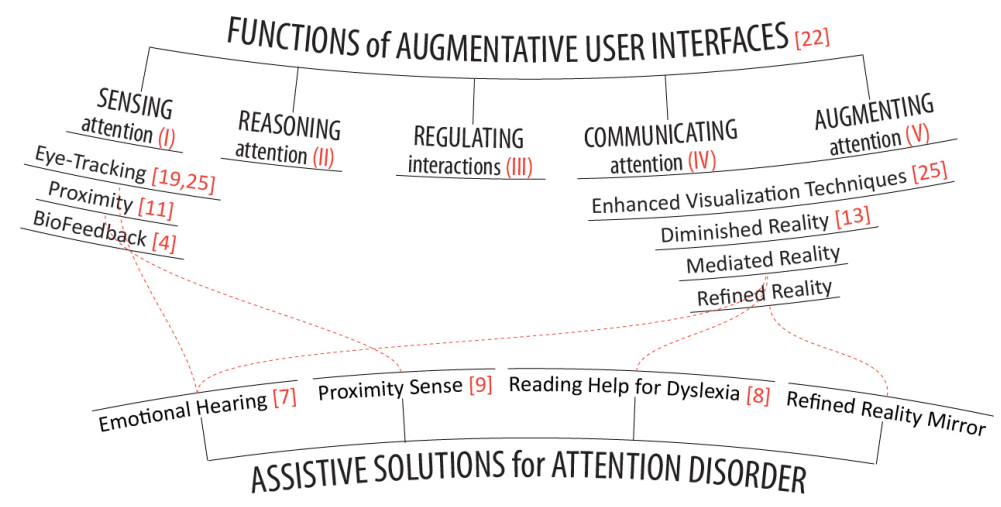

Figure 2: Classification of AUI functions for related techniques in the field and matching these techniques with assistive solutions for different attention disorder situations

(Fig. 2). All of these studies offer a backdrop for our diminished reality concept.

From a filtering method perspective, Herling [13] developed an algorithm to remove unwanted shapes or objects from livecaptured images and called this diminished reality. Another example of a similar image-filtering algorithm is a real-time foveation filter that again uses eye-gaze and blurs the unattended vision [20]. It is obvious that head-up or head-mounted augmented reality technologies such as large see-through displays will soon use these different filtering techniques for removing irrelevancies or highlighting relevant visual information before it reaches our eye. We investigate solutions for diminished reality systems, starting with a case study on individuals with ASD.

\section{Interactive Assistive Technologies for Individuals with Attention Disorder}

The previously discussed studies on AUIs aim to help ameliorate workplace attention problems. We are all affected by disabling situations but to a lesser extent than individuals with particular perception and cognition structures, such as the ones diagnosed with dyslexia, ASD, ADHD, and so on. It is claimed that dyslexic individuals suffer from not being able to select information effectively rather than mis-encoding [8]. By using the information that a dyslexic individual's visual awareness can receive limited amount of the encoded information by the eye, Roach [21] suggested that filtering the irrelevant visual information before it reaches the eye would help. He examined searching and filtering functions used while reading and reached some valuable information such as the positive effect of cueing.

It is known that technology can help the education, rehabilitation and the daily life process of individuals with ASD. While attention-challenged individuals suffer from disabilities in communication, socialization and imagination, some autistics are good with structural information processing [1] and focusing on specific things. This makes them potentially good users of machines and technology [3]. Some other facts about individuals with ASD are that they have better visual than auditory perception [10], and different types of autistics have different visual representation abilities (objects, realistic image, realistic illustrations, text). All of these facts are significant clues for an interactive solution aimed at the varied visual needs of ASD individuals. Altogether, considerable work has been focused on technological solutions for improving the quality of life for autistics using mechanical and visual abilities. Leaving the rehabilitation and educational methods apart, daily life solutions mostly concentrate on helping with communication abilities.

ASD individuals lack abilities of understanding facial expressions, cause-effect reasoning, eye-contact communication and abstract language. These facts cause social communication problems. Augmentative Alternative Communication (AAC) technologies are one method which uses visual or auditory messages to augment and regulate the intended communication. The emerging technologies in wearables, robotics, and ubiquitous computing have high potential for AAC studies. Now we have the technological ability to process the emotional state of our surroundings and help individuals understand emotions. In one promising project, Kaliouby \& Robinson [14] suggest a prosthetic solution; the "Emotional Hearing Aid"; a system that watches facial expressions and gives the information to the ASD individual, although this possible solution must overcome the difficulty that most ASD individuals do not like attachments. New technologies also bring proximity information into consideration for socially disabled individuals. Feil-Seifer \& Mataric [9] used proximal information gathered through the observation of autistics and robots. Examining these studies, we hypothesize that interactive systems for individuals with ASD is a promising topic within the area of interactive media design. Even though it may seem obvious that AUIs can help ASD-challenged individuals, this is still an open issue. We also believe that such an experience will lead to significant results for different approaches in human factor research.

\section{METHOD}

The motivation we have summarized above encouraged us to further explore possible AUI solutions for enhancing everyday life process of individuals with ASD. With this perspective, we started examining interactive technologies for individuals with ASD, the fields of AUI and augmentation of visual attention. Examining the relevancy of AUI for ASD rounded off the literature review.

After the extensive literature overview we organized eight design workshops (Fig. 3) with interaction designers and ASD domain experts (rehabilitators, caretakers, and family members of individuals with ASD) who will also be potential users of such solutions when accompanying an ASD individual. During a period of two months, we carried out eight sequential workshops. For each workshop we invited new participants; always five interaction designers and two ASD experts. At the start of each workshop we briefed the participants with insights from our literature review. These workshops benefited our exploration by 
examining novel ways of bringing our research problem closer to current HCI solutions.

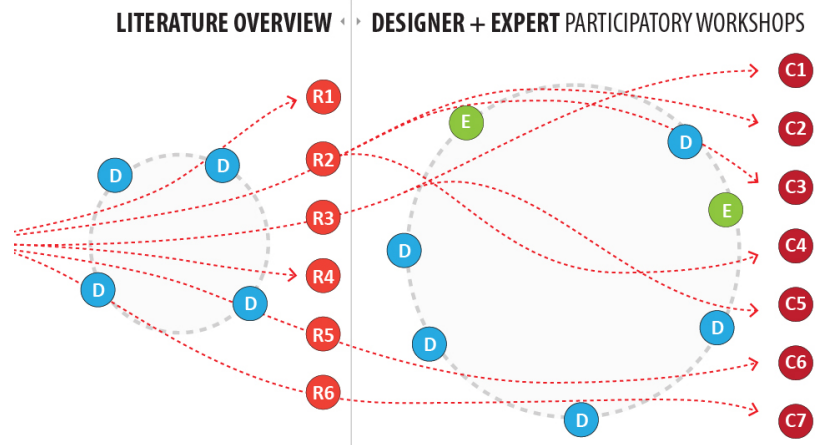

Figure 3: Exploration methodology: Literature overview (left) followed by participatory design workshops (right). Designers (D) and domain experts $(E)$ work together to explore design requirements $(R)$ and criteria $(\mathrm{C})$.

\section{DISCUSSIONS AND CONCEPTS}

First we carried out a literature review of human-factor related aspects of AUIs. The insights gathered are presented next to designers of AUIs as targeted readership:

R1. In most HCI uses, the environment "disables" the user. Human Factor research deals with the subject with an approach claiming that better interaction can be achieved by considering disabling environment factors.

R2. Particular individuals with attention disorder are even more affected by distractors in their environment, even in their everyday life situations without using technology.

R3. There are interactive assistive communication solutions for ASD individuals to help them with education. But not many of these studies try to find everyday life solutions.

R4. These individuals are sometimes good with technology and mechanical abilities. Interactive technologies can make their life easier. This is also a valuable case study for interactive media design.

R5. AUI is one paradigm that can help us build smart systems watching our needs and regulating the interaction in both disabled conditions and information bombardment situations.

R6. DR is an emerging technique to optimize communication in HCI.

In a second step, we used the six requirements (R1-R6) as input for two design workshops targeted at overcoming attention problems of individuals with ASD in their everyday lives. Evaluating these requirements, the workshops generated a set of seven criteria (C1-C7):

C1. ASD individuals have problems in perception and attention that leads to impairment in social communication. Assistive technologies can be used to optimize communication in their everyday life.

C2. They confront many distractors in their everyday life. Sometimes they dislike specific colors, objects, or people. Filtering out irrelevant information might help with augmenting attention.
C3. They spend most of their time in classrooms, living rooms or in room-like offices and prefer sameness in environments. Therefore, the solution should help with organizing the information in their space.

C4. These individuals do not like attachments to their bodies. The solution should not be a prosthetic solution like a headmounted augmented reality device. It can be a see-through or reflecting plane.

C5. ASD individuals cannot understand mental states, facial expressions, abstract meanings of messages, or causeeffect relationships. Assistive technology can optimize these expressions and messages so that they can be perceived.

C6. ASD individuals do not like eye contact with other people. The solution might help communication without the need for eye contact. It is preferable to stay away from gazecontingent interaction techniques.

C7. Different ASD individuals might have different perceptual abilities. For instance, some might be better than others with visual, auditory or verbal messages. Systems should support multi-modal communication abilities.

Based on these discussions, we came up with several different ideas on how an assistive AUI technology could be designed to overcome social communication and presence problems of an ASD individual. These studies led us to the question; why not build a space where the information is projected onto surfaces and things (Fig. 1)? Such a system would keep track of the state of the room and needs of the user, and use DR techniques like filtering irrelevant information to augment the user's attention depending on given sets of information. We believe that this might be a natural way of assisting communication without adding barriers. In detail, we explored the concept regarding content filtering and guidance (Figs. 4-5), reflecting proxemics information (Figs. 6-7) and spatial design (Fig. 8).

In terms of content filtering (Fig. 4), the "mirror-wall" concept can (a) filter out irrelevant contents by which an ASD individual is affected in the work environment, (b) reflect the irrelevant contents as if they are relevant, (c) only reflect the relevant contents which the ASD individual needs or wants, or (d) only reflect the contents which guide the ASD individual to carry out specific actions. The latter function is illustrated in detail in Fig. 5 that shows the mirror-like wall guiding ASD individual to perform next steps of his/her actions in a personal or social setting such as clearing the table or greeting someone. 

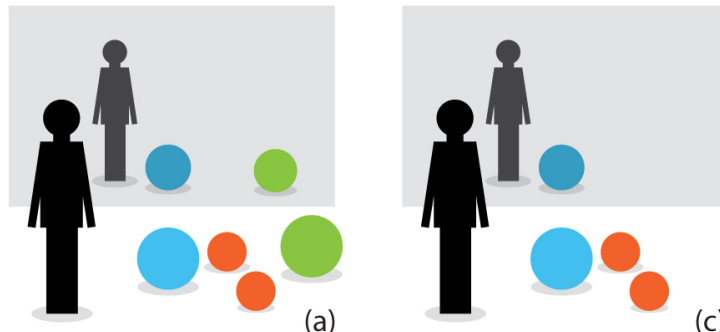

(c)
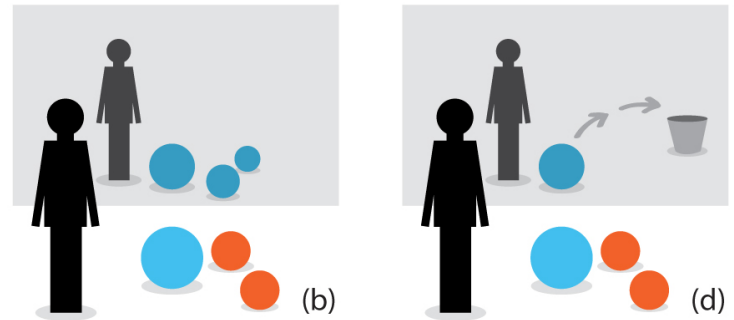

Figure 4: Content filtering alternatives (a-d)
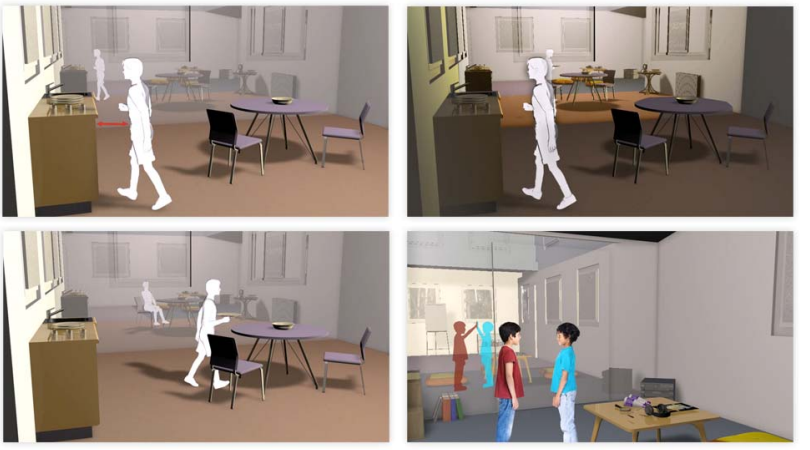

Figure 5: Content guidance on the mirror wall

One other discussion has been to overcome proxemics related problems. Individuals with ASD suffer from social presence within an environment. They can be distracted by the presence of a person, the distance between her/himself and others, people's intimate gestures or physical interactions. Our proposed AUI concept deals with this issue by augmenting the space by projecting information onto the space (Fig. 6). The attentive space can show the ASD individual's tolerance area (Fig. 7b) in order to support (or block, if needed) the communication with surroundings mutually by using the position and proximity information of each individual in the working area. This solution can help an ASD individual to know who is getting closer.

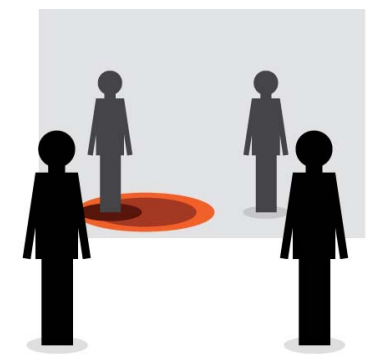

Figure 6: Proxemics information on the mirror
A second concept can be to reflect the information only to the other user and not to the individual with ASD, such as when the proximity related information is augmented as the shadow of the individual with ASD (Fig. 5a) or a surface in the environment, which cannot be seen by individuals with ASD (Fig. 7c).

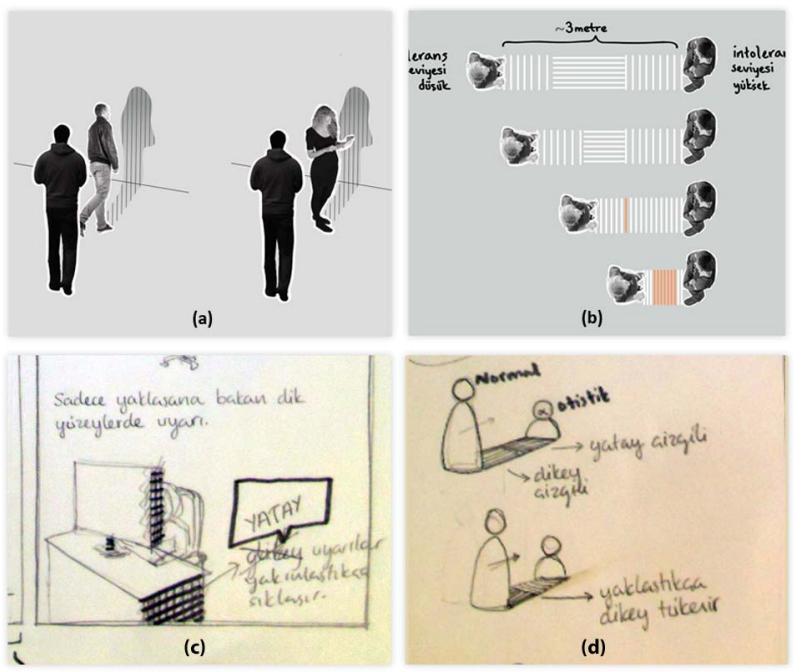

Figure 7: Proxemics information on the mirror

As a last critical point, the attentive space's spatial design (Fig. 8) can be applied to several different compositions in the surroundings; (a) one wall as well as (b) more than one in the ASD individual's working area in order to minimize the possible interruptions; (c) the eye level so that the ASD individual interacts with it only when he stands and sits; or (d) the ASD individual's desk as a separator.
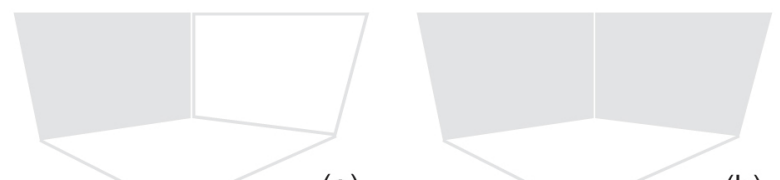

(a)

(b)
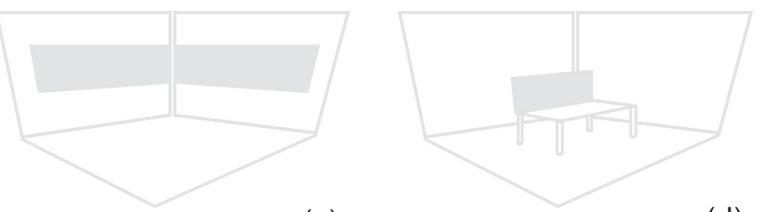

(c)

(d)

Figure 8: Placement alternatives (a-d) for mirror-like or see-through surface

\section{CONCLUSION}

Exploring this experimental and inductive yet promising conceptual idea of an assistive interactive technology, we have presented a DR approach, targeting enhancement of attention and communication disorder challenges of ASD individuals in their daily life conditions. We have presented expected future applications of DR and discussed related human factors. As a result of eight participatory design workshops, we came up with some design solutions of an interactive DR space with surfaces that filter out irrelevant visual data present in the environment 
before it reaches the eye, or augmenting the surroundings by projecting assistive information onto different types of surfaces. We believe that this study will contribute in two ways; (a) in terms of AUI research with some exploratory ideas of using different surfaces and concepts (mirror-like wall, one-way visible surfaces, shared view surfaces), (b) and in terms of new possible research directions for the use of interactive technologies for improved life quality or experience for individuals with ASD. The ideas presented here should be further studied within prototype-based user studies for alternative use cases, scenarios, and user categories.

\section{ACKNOWLEDGMENTS}

For artwork in Fig. 4 we thank Ya mur Akın Karagöz; for artwork in Fig. 5 we thank Damla Çay.

\section{REFERENCES}

1. Baron-Cohen, S., Wheelwright, S., Lawson, J., Griffin, R., Hill, J. The Exact Mind: Empathizing and Systemizing in Autism Spectrum Conditions. In Goswami, U, (ed) Handbook of Cognitive Dev. 2004

2. Basu S. \& Pentland A. Smart Headphones. In Extended Abstracts of CHI'01. Seattle: ACM Press, 2001

3. Cafiero, J. Meaningful Exchanges for People with Autism: An Introduction to Augmentative \& Alternative Communication. Bethesda, MD: Woodbine House,

4. Chen, D., \& Vertegaal, R. Using mental load for managing interruptions in physiologically attentive userinterfaces. In Proceedings of CHI 2004 pp. 1513-1516. Vienna: ACM Press.

5. Danninger, M., Vertegaal, R., Siewiorek, D., \& Mamuji, A. Using social geometry to manage interruptions and co-worker attention in office environments. In Proceedings of Graphics Interface 2005.

6. De Korte, E., Kuijt-Evers, L., \& Vink, P., Effects of the office environment on health and productivity 1 : auditory and visual distraction. Ergonomics and Health Aspects of Work with Computers. Proceedings International Conference, EHAWC 2007. Held as Part of HCI International 2007, 26e33.

7. De Vincenzi, A., Yao, L., Ishii, H., \& Raskar, R. Kinected conference: augmenting video imaging with calibrated depth and audio. In Proceedings of the ACM 2011 conference on Computer supported cooperative work (CSCW '11). ACM, New York, NY, USA, 621-624.

8. Facoetti A, Molteni M. The gradient of visual attention in developmental dyslexia. Neuropsychologia 2001; 39: 352-7.

9. Feil-Seifer, D. J., M. J. Matari. Using proxemics to evaluate human-robot interaction. In Proceedings of the International Conference on Human-Robot Interaction, Osaka, Japan, Mar 2010

10. Goodwin, M.S. Enhancing and accelerating the pace of autism research and treatment: The promise of developing innovative technology. Focus on Autism and Other Developmental Disabilities, 23, 200

11. Greenberg, S., Marquardt, N., Ballendat, T., Diaz-Marino, R., Wang, M. Proxemic interactions: the new ubicomp?. interactions 18, 1, 42-50, 2011
12. Griswold, D.E., Barnhill, G.P., Myles, B.S., Hagiwara, T., \& Simpson, R.L. Asperger Syndrome and Academic Achievement Focus Autism Other Dev Disabl 2002 17: 94

13. Herling, J. \& Broll, W. Advanced self-contained object removal for realizing real-time Diminished Reality in unconstrained environments Mixed and Augmented Reality (ISMAR), 2010 9th IEEE International Symposium Ilmenau Univ. of Technol., Ilmenau, Germany pp. 207 - 212 13-16 Oct. 2010

14. Kaliouby, R., Robinson,P. Real-Time Inference of Complex Mental States from Facial Expressions and Head Gestures. In the IEEE International Workshop on Real Time Computer Vision for Human Computer Interaction at CVPR, 2004

15. Liebl A., Haller, J., Jödicke, B., Baumgartner, H., Schlittmeier, S., Hellbrück, J.; Combined effects of acoustic and visual distraction on cognitive performance and well-being, Applied Ergonomics, 43 (2912) 424-434

16. Mueller F. \& Karau M.: Transparent Hearing. In Extended Abstracts of CHI'02, ACM Press, 2002.

17. Narayanan, N. H. \& Yoon, D. Reactive Information Displays. In Proceedings of INTERACT 2003: Ninth IFIP TC 13 International Conference on Human-Computer Interaction, IOS Press, 244-251

18. Parkhurst, D., Culurciello, E., \& Niebur, E. Evaluating variable resolution displays with visual search: task performance and eye movements. In Proceedings of the eye tracking research and applications symposium (pp. 105-109). New York, USA: ACM Press, 2000

19. Pederson, T., Hansen, W., Mardanbeigi, D. Investigations of the Role of Gaze in Mixed-Reality Personal Computing. In Proceedings of the 2nd Workshop on Eye Gaze in Intelligent Human Machine Interaction held at IUI'2011, Stanford, February 13

20. Perry, J. S., \& Geisler, W. S. Gaze-contingent real-time simulation of arbitrary visual fields. In B. E. Rogowitz \& T. N. Pappas (Eds.), Human vision and electronic imaging VII, SPIE-4662 (pp. 57-69). Bellingham, WA, USA: The International Society for Optical Engineering. 2002

21. Roach, N.W. and Hogben, J.H. Impaired filtering of behaviourally irrelevant visual information in dyslexia. Brain 130, 771-785, 2007

22. Shell, J.S., Vertegaal, R., \& Skaburskis, A.W. EyePliances: attention-seeking devices that respond to visual attention. In Proceedings of the conference on human factors in computing systems (pp. 770-771). New York, USA: ACM Press, 2003

23. Skaburskis, A.W., Vertegaal, R., \& Shell, J.S. Auramirror: reflections on attention. In Proceedings of the 2004 symposium on Eye tracking research \& applications (ETRA '04). ACM, New York, NY, USA, 101-108.

24. Vertegaal, R., Shell, J.S., Chen, D., Mamuji, A. Designing for augmented attention: Towards a framework for attentive user interfaces Computers in Human Behavior 22, 771-789, 2006

25. Toet, A. Gaze directed displays as enabling technology for attention aware systems. Computers in Human Behavior 22, 615-647, 2006 